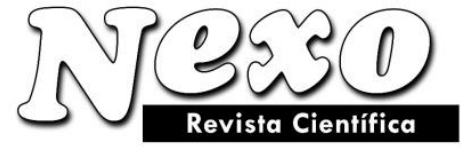

Vol. 34, No. 04, pp. 1301-1321/Septiembre 2021
ISSN-E 1995-9516

Universidad Nacional de Ingeniería COPYRIGHT @ (UNI). TODOS LOS DERECHOS RESERVADOS http://revistas.uni.edu.ni/index.php/Nexo https://doi.org/10.5377/nexo.v34i04.12666

\title{
Application of bayesian scientific approach to constructing the statistical estimations for solving metrological and measurement problems
}

\section{Aplicación del enfoque científico bayesiano a la construcción de estimaciones estadísticas para resolver problemas metrológicos y de medición}

Khayrullin Rustam Zinnatullivich ${ }^{*}$, Khaimuldinova Altyngul Kumashevna ${ }^{2}$, Taimanova Gulnara Kabzhanovna $^{2}$, Sarsembayeva Tolkyn Erzhanovna ${ }^{3}$, Volkov Vladimir Sergeevich ${ }^{4}$, Shamina Svetlana Vladimirovna ${ }^{5}$, Lyalin Evgenij Aleksandrovich ${ }^{6}$, Kabanov Oleg Vladimirovich ${ }^{7}$

${ }^{1}$ Moscow State University of Civil Engineering, Yaroslavskoe shosse 26, 129337, Moscow, Russian Federation, Department of Applied Mathematics

${ }^{2}$ L.N. Gumilyov Eurasian National University, 13 Kazhymukan St., Nur-Sultan, Kazakhstan, Z01C0X0

${ }^{3}$ S. Seifullin Kazakh Agrotechnical University, Zhenis avenue, 62, Nur-Sultan, Kazakhstan, 010011

${ }^{4}$ Voronezh State University of Forestry and Technologies named after G.F. Morozov

2/1 Lesokulturny lane, Voronezh, 394087, Russia

${ }^{5}$ South Ural State Agrarian University, Troitsk, Chelyabinsk region, Gagarin str., 13, 457100

${ }^{6}$ Perm State Agro-Technological University, 113, Geroev Khasana Street, Perm, Russia, 614025

${ }^{7}$ Ogarev Mordovia State University, Russia, Republic of Mordovia, Saransk, 430005, bolshevitskaya street 68

(recibido/received: 21-mayo-2021; aceptado/accepted: 15-agosto-2021)

Corresponding author: ku.academic@yahoo.com

\begin{abstract}
Nowadays, constructing effective statistical estimates with a limited amount of statistical information constitutes a significant practical problem. The article is devoted to applying the Bayesian scientific approach to the construction of statistical estimates of the parameters of the laws of distribution of random variables. Five distribution laws are considered: The Poisson law, the exponential law, the uniform law, the Pareto law, and the ordinary law. The concept of distribution laws that conjugate with the observed population was introduced and used. It is shown that for considered distribution laws, the parameters of the laws themselves are random variables and obey the typical law, gamma law, gamma - normal law, and Pareto law. Recalculation formulas are obtained to refine the parameters of these laws, taking into account posterior information. If we apply the recalculation formulas several times in a row, we will get some convergent process. Based
\end{abstract}


on a converging process, it is possible to design a process for self-learning a system or self-tuning a system. The developed scientific approach was applied to solve the measuring problems for the testing measuring devices and technical systems. The results of constructing point estimates and constructing interval estimates for these laws' parameters are given. The results of comparison with the corresponding statistical estimates constructed by the classical maximum likelihood method are presented.

Keywords: measurement accuracy, Bayesian scientific approach, a posteriori information, metrological and measurement problems.

\section{RESUMEN}

Hoy en día, la construcción de estimaciones estadísticas efectivas con una cantidad limitada de información estadística constituye un problema práctico significativo. El artículo está dedicado a aplicar el enfoque científico bayesiano a la construcción de estimaciones estadísticas de los parámetros de las leyes de distribución de variables aleatorias. Se consideran cinco leyes de distribución: la ley de Poisson, la ley exponencial, la ley uniforme, la ley de Pareto y la ley ordinaria. Se introdujo y utilizó el concepto de leyes de distribución que se conjugan con la población observada. Se muestra que para las leyes de distribución consideradas, los parámetros de las leyes mismas son variables aleatorias y obedecen la ley típica, la ley gamma, la ley gamma normal y la ley de Pareto. Se obtienen fórmulas de recálculo para afinar los parámetros de estas leyes, teniendo en cuenta información posterior. Si aplicamos las fórmulas de recálculo varias veces seguidas, obtendremos algún proceso convergente. A partir de un proceso convergente, es posible diseñar un proceso para el autoaprendizaje de un sistema o el autoajuste de un sistema. El enfoque científico desarrollado se aplicó para resolver los problemas de medición de los dispositivos de medición de prueba y los sistemas técnicos. Se dan los resultados de la construcción de estimaciones puntuales y la construcción de estimaciones de intervalo para los parámetros de estas leyes. Los resultados de la comparación con el estadístico correspondiente

Palabras clave: precisión de la medición, enfoque científico bayesiano, información a posteriori, problemas metrológicos y de medición.

\section{INTRODUCTION}

The problem of constructing effective statistical estimates is an important applied task. This problem has to be solved when conducting scientific research related with processing of statistical data in areas such as biochemistry, machine vision problem, automated digital control problem, the development and implementation of the concept of a smart home - a smart city, problem of economical simulation, for the purpose of development and implementation of new technologies in manufacture (Vishnyakov \& Egorov, 2013: Duyguİçen, 2019: Yang et al, 2018: Higgins et al, 2019: Touzani et al, 2019: \& Butenweg, 2019).

In (Ayvazyan, 2008). the general scheme of the Bayesian approach in statistical estimation in econometrics was described. Some other applied problem of applications of Bayesian scientific 
approach including the construction and investigation of self-leaning systems and development of pattern recognition problem are described in (Kropotov, \& Vetrov, 2007: Yochan \& Jinkyun, 2019, Wang et al, 2019: Geweke \& Durham, 2019: Michael et al, 2018).

Investigations described in there were devoted to practical problems of construction of effective statistical estimation when processing measurement results, when setting and studying measurement problems, and when processing the results of tests of measuring equipment. In the Bayesian approach is described in the problems of constructing statistical estimates in case when the studied statistical sampling of data has a normal distribution law. However, for other distribution laws in solving metrological problems, the Bayesian approach has not yet been developed and used (Lavrik et al, 2019: Francisco et al, 2019: Gao et al, 2019: Mishchenko et al, 2021: Lazarenko et al, 2021).

This article describes a method for constructing statistical parameter estimates for solving measurement problems and processing test results for five fairly common distribution laws: Poisson's law, exponential law, uniform law, Pareto law with unknown shape parameter and known shift parameter and normal law in two cases: as with unknown means and known dispersion, as unknown means and unknown dispersion. The concept of distributions conjugated with the observed population is introduced and used. The method for calculating specific values of the parameters of conjugated distribution laws was developed. Algorithm for correcting these parameters is described with joint consideration of a priori and posterior information was created. Examples of constructing of point estimates and of interval estimates are presented (Khayrullin et al, 2021: Volchkov et al, 2019: Khayrullin, 2019).

\section{MATERIALS AND METHOD}

\section{General scheme of the Bayesian approach in statistical estimation}

Let the distribution law of the analyzed random variable depends on $S$-dimensional vector of numerical parameters $\Theta=\left(\theta_{1}, \theta_{2}, \ldots, \theta_{s}\right)$. Here capital letter is used to denote vector, and small letters are used to denote scalar quantities. The problem is to construct the best, in a certain sense, statistical estimation $\hat{\Theta}$ of this vector of parameters on the base of available statistical sampling $X=\left(x_{1}, x_{2}, \ldots, x_{n}\right)$. Here capital letter is used to denote statistical data in general and small letters are used to denote specific realizations of a random variable.

The general logic scheme of the Bayesian scientific approach in statistical estimation procedure is presented in Figure1. 


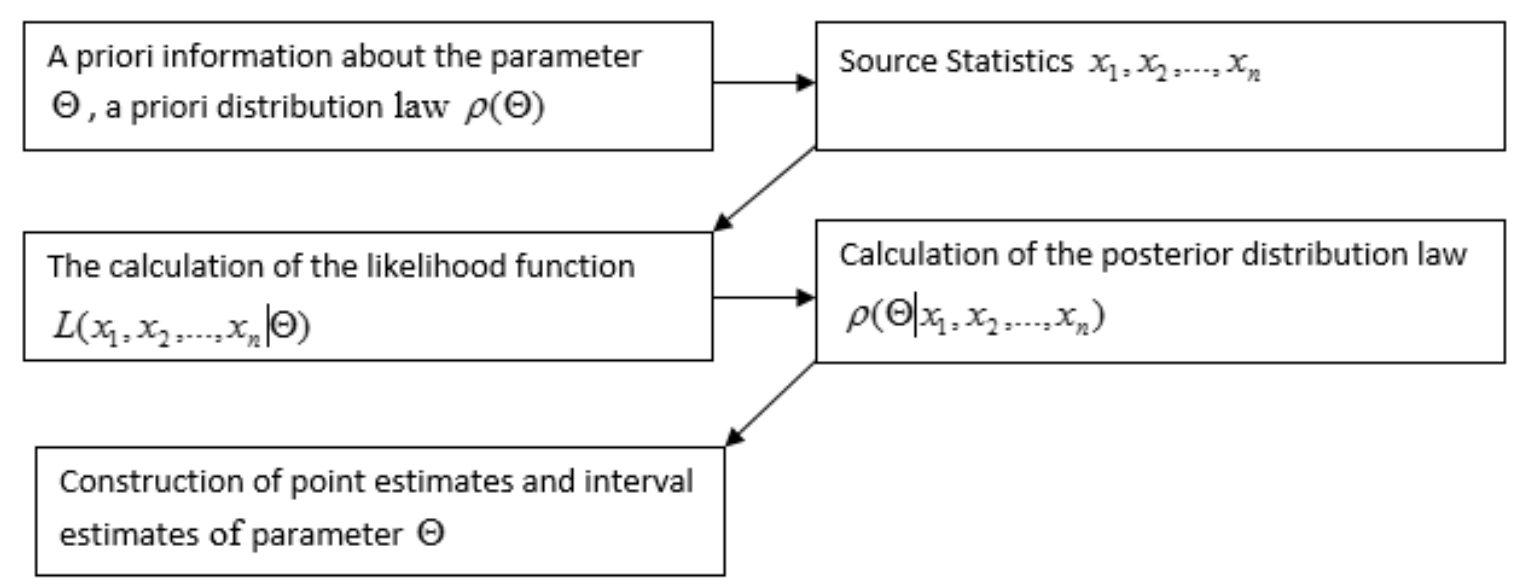

Figure1. General logical scheme of the Bayesian approach in statistical estimation

Let us describe the implementation of the Bayesian estimation scheme for the components of vector of parameter $\Theta$. A priori information about the vector of parameters is based on the background of the functioning of the analyzed process and on expert evaluations of its essence and specificity too. We assume that a priori information is given by means of function of the distribution density $\rho(\Theta)$.

Statistical data $X=\left(x_{1}, x_{2}, \ldots, x_{n}\right)$ are generated in accordance with the law of probability distribution $f(X \mid \Theta)$, where $f(X \mid \Theta)$ - the meaning of the density function of the observed random variable $\xi=\left(\xi^{(1)}, \xi^{(2)}, \ldots, \xi^{(k)}\right)$ at a point $X=\left(x_{1}, x_{2}, \ldots, x_{n}\right)$ is understood. By default, it is assumed that statistics sampling $x_{1}, x_{2}, \ldots, x_{n}$ with a fixed one $\Theta$ are statistically independent and its form a random sample from the analyzed set. So, getting new statistical data, we attach the corresponding sample (empirical) information to the available a priori information about the parameter (as a function $\rho(\Theta)$ ). Accordingly to maximum likelihood method (MLM), the conditional likelihood function $L\left(x_{1}, x_{2}, \ldots, x_{n} \mid \Theta\right)$ for each given value of $\Theta$ the available observations $x_{1}, x_{2}, \ldots, x_{n}$ is determined by the formula:

$$
L\left(x_{1}, x_{2}, \ldots, x_{n} \mid \Theta\right)=f\left(x_{1} \mid \Theta\right) \cdot f\left(x_{2} \mid \Theta\right) \cdot \ldots \cdot f\left(x_{n} \mid \Theta\right)
$$

The calculation of the posterior distribution $\tilde{\rho}\left(\theta \mid x_{1}, x_{2}, \ldots, x_{n}\right)$ marked by a wave from above is carried out using the Bayes formula:

$$
\tilde{\rho}\left(\Theta \mid x_{1}, x_{2}, \ldots, x_{n}\right)=\frac{\rho(\Theta) L\left(\left|x_{1}, x_{2}, \ldots, x_{n}\right| \Theta\right)}{\int L\left(\left|x_{1}, x_{2}, \ldots, x_{n}\right| \Theta\right) \rho(\Theta) d \Theta}
$$

The construction of Bayesian point estimates and interval estimates is based on knowledge of the posterior distribution $\tilde{\rho}\left(\theta \mid X_{1}, X_{2}, \ldots, X_{n}\right)$ given by relation (2). In particular, the average value of this distribution is used as Bayesian point estimates: 


$$
\hat{\Theta}_{C P}^{(B)}=E\left(\Theta \mid x_{1}, x_{2}, \ldots, x_{n}\right)=\int \Theta \tilde{\rho}\left(\Theta \mid x_{1}, x_{2}, \ldots, x_{n}\right) d \Theta .
$$

Note that to determine the general form of a posteriori density $\tilde{\rho}\left(\theta \mid x_{1}, x_{2}, \ldots, x_{n}\right)$ it is enough for us to know only the numerator of the right-hand side of (2), since the denominator of this expression plays the role of a normalizing factor and does not depend on $\Theta$. This fact greatly simplifies the process of practical construction of statistical estimates.

We also note one important optimal property of the estimate $\hat{\Theta}_{C P}^{(B)}$. Let $\hat{\Theta}\left(x_{1}, x_{2}, \ldots, x_{n}\right)$ be some estimate of the parameter $\Theta$. It is shown that if the quality of the assessment of $\hat{\Theta}\left(x_{1}, x_{2}, \ldots, x_{n}\right)$ we measure by a posteriori Bayesian risk

$$
R^{(E)}\left(x_{1}, \ldots, x_{n}\right)=E\left(\left(\hat{\Theta}\left(x_{1}, \ldots, x_{n}\right)-\Theta\right)^{2} \mid x_{1}, \ldots, x_{n}\right)=\int\left(\hat{\Theta}\left(x_{1}, \ldots, x_{n}\right)-\Theta\right)^{2} \tilde{\rho}\left(\Theta \mid x_{1}, x_{2}, \ldots, x_{n}\right) d \Theta
$$

or its value averaged over all possible samples $R_{C P}^{(5)}$, then the Bayesian estimate (3) is the best.

To construct a Bayesian confidence interval for the vector of parameters $\Theta$, it is necessary to calculate, according to formula (2), the function $\tilde{\rho}\left(\theta \mid x_{1}, x_{2}, \ldots, x_{n}\right)$ of the posterior distribution law of the parameter $\Theta$, and then, using the given confidence probability $P_{0}$, determine the $0,5 \cdot\left(100+P_{0}\right) \%$ and $0,5 \cdot\left(100-P_{0}\right) \%$ points of this law, which give the left and right ends of the desired interval estimate, respectively

\section{A priori distribution laws conjugating with the observed general statistical sampling}

Below we give the definition of the conjugate distribution law. A set of a priori distribution laws $G=\{\rho(\Theta) ; D\}$ are called conjugate with respect to the investigated general statistical data with density function $f(X \mid \Theta)$ (or with respect to the likelihood function $L\left(x_{1}, x_{2}, \ldots, x_{n} \mid \Theta\right)$, if the posterior distribution law $\tilde{\rho}\left(\Theta \mid x_{1}, x_{2}, \ldots, x_{n}\right)$ calculated by formula (2) again belongs to the same set $G=\{\rho(\Theta) ; D\}$. Here $D$ - is the value of the vector of parameters of the distribution laws $\rho(\Theta)$

In other words, a set of distributions $G=\{\rho(\Theta) ; D\}$ is conjugate to $L\left(x_{1}, x_{2}, \ldots, x_{n} \mid \Theta\right)$ if it is closed with respect to operation (2) of converting a priori distribution law to the posterior one.

Thus, the use of probability distributions conjugate with respect to $L$ laws as a priori laws makes it necessary only to recalculate the numerical values of the parameters of the known distribution law in the transition from a priori distribution law to a posterior distribution law.

\section{A condition of the existence of a conjugate set of a priori distribution laws.}

If the likelihood function $L\left(x_{1}, x_{2}, \ldots, x_{n} \mid \Theta\right)$ can be represented in the form :

$$
L\left(x_{1}, x_{2}, \ldots, x_{n} \mid \Theta\right)=v\left(T_{1}\left(x_{1}, x_{2}, \ldots, x_{n}\right), \ldots, T_{m}\left(x_{1}, x_{2}, \ldots, x_{n}\right) ; \Theta\right) \cdot \psi\left(x_{1}, x_{2}, \ldots, x_{n}\right),
$$


where $T_{j}\left(X_{1}, X_{2}, \ldots, X_{n}\right),(j=1,2, \ldots, m)$ and $\psi\left(x_{1}, x_{2}, \ldots, x_{n}\right)$ - are some functions of statistical sampling $x_{1}, x_{2}, \ldots, x_{n}$ that are independent for each value of the vector of parameter $\Theta$, then there exists a set $G=\{\rho(\Theta) ; D\}$ of a priori distribution laws a conjugating with $L\left(x_{1}, x_{2}, \ldots, x_{n} \mid \Theta\right)$

Now we provide that the conditions for the existence of a conjugate a priori distribution are satisfied for all the distribution laws considered above.

\section{Poisson distribution:}

$$
f(x \mid \theta)=P\{\xi=x \mid \theta\}=\frac{\theta^{x}}{x !} e^{-\theta}, x=1,2,3, \ldots,
$$

$\theta$ - unknown value for parameter,

$$
L\left(x_{1}, x_{2}, \ldots, x_{n} \mid \theta\right)=\prod_{i=1}^{n} \frac{\theta^{x_{i}}}{x_{i} !} e^{-\theta}=e^{-n \theta} \cdot \theta^{\sum_{i=1}^{n} x_{i}} \cdot \prod_{i=1}^{n} \frac{1}{x_{i} !} .
$$

We have: $m=1, T\left(x_{1}, x_{2}, \ldots, x_{n}\right)=\sum_{i=1}^{n} x_{i}=\bar{x}$.

\section{Exponential distribution:}

$$
f(x \mid \theta)=\left\{\begin{array}{ll}
\theta e^{-\theta x} & , \quad x \geq 0 \\
0 & , \quad x<0
\end{array},\right.
$$

$\theta$ - unknown value of parameter,

$$
L\left(x_{1}, x_{2}, \ldots, x_{n} \mid \theta\right)=\left\{\begin{array}{ll}
\theta^{n} e^{-\left(\sum_{i=1}^{n} x_{i}\right) \theta} & , \quad x \geq 0 \\
0 & , \quad x<0
\end{array} .\right.
$$

In this case, we have: $m=1, T\left(x_{1}, x_{2}, \ldots, x_{n}\right)=\sum_{i=1}^{n} x_{i}=\bar{x}$.

Distribution uniform on a closed interval $[0 ; \Theta]$ :

$$
f(x \mid \theta)=\left\{\begin{array}{ll}
\frac{1}{\theta} & , \quad 0 \leq x \leq \theta \\
0 & , \quad x \notin[0 ; \theta]
\end{array},\right.
$$

$\theta$ - unknown value of parameter,

$L\left(x_{1}, x_{2}, \ldots, x_{n} \mid \theta\right)=\left(\frac{1}{\theta}\right)^{n}$ for $\theta \geq x_{\max }(n)=\max _{1 \leq i \leq n} x_{i}$

In this case, we have: $m=1 ; T\left(x_{1}, x_{2}, \ldots, x_{n}\right)=x_{\max }(n)$.

Pareto distribution with an unknown shape parameter value $\theta$ and a known shift value $x_{0}$ : 


$$
f(x \mid \theta)= \begin{cases}\frac{\theta x_{0}^{\theta}}{x^{\theta+1}} & , \quad x \geq x_{0} \\ 0 & , \quad x<x_{0}\end{cases}
$$

Respectively:

$$
L\left(x_{1}, x_{2}, \ldots, x_{n} \mid \theta\right)=\theta^{n} x_{o}^{n \theta} \cdot\left(\prod_{i=1}^{n} x_{i}\right)^{-(\theta+1)}=\theta^{n}\left(\frac{g_{n}}{x_{0}}\right)^{-n \theta} \cdot g_{n}^{-n},
$$

here $g_{n}=\left(\prod_{i=1}^{n} x_{i}\right)^{\frac{1}{n}}$ - geometric mean value of statistical sampling of analyzed random variable $x_{1}, x_{2}, \ldots, x_{n}$. So, we have $m=1, T\left(x_{1}, x_{2}, \ldots, x_{n}\right)=g(n)$.

Normal distribution law with known dispersion $\sigma_{0}^{2}$ :

Let random value $\xi$ has the normal distribution law $\xi \in N\left(\theta, \sigma_{0}^{2}\right)$.

Respectively:

$$
\begin{aligned}
& L\left(x_{1}, x_{2}, \ldots, x_{n} \mid \theta\right)=\prod_{i=1}^{n} f\left(x_{i} \mid \theta\right)=\left(\frac{1}{\sqrt{2 \pi} \sigma_{0}}\right)^{n} \exp \left(-\frac{1}{2 \sigma_{0}^{2}} \sum_{i=1}^{n}\left(x_{i}-\theta\right)^{2}\right)= \\
& \quad=\exp \left(-\frac{1}{2 \sigma_{0}^{2}} \sum_{i=1}^{n}(\bar{x}-\theta)^{2}\right) \cdot\left(\frac{1}{\sqrt{2 \pi} \sigma_{0}}\right)^{n} \cdot \exp \left(-\frac{1}{2 \sigma_{0}^{2}} \sum_{i=1}^{n}\left(x_{i}-\bar{x}\right)^{2}\right) .
\end{aligned}
$$

So we have $m=1, T\left(X_{1}, X_{2}, \ldots, X_{n}\right)=\bar{x}=\frac{1}{n} \sum_{i=1}^{n} x_{i}$.

Normal distribution with unknown means $\theta_{1}$ and unknown parameter $\theta_{2}=1 / \sigma$ (unknown dispersion $\left.D=\sigma^{2}=\left(1 / \theta_{2}\right)^{2}\right)$ :

Let random value $\xi$ has the normal distribution $\xi \in N\left(\theta_{1} ; 1 / \theta_{2}\right)$.

Respectively: $m=2 ; T_{1}\left(X_{1}, X_{2}, \ldots, X_{n}\right)=\bar{x} ; T_{2}\left(X_{1}, X_{2}, \ldots, X_{n}\right)=s^{2}=\frac{1}{n} \sum_{i=1}^{n}\left(x_{i}-\bar{x}\right)^{2}$.

Thus, for each considered distribution laws, there is an a priori distribution law conjugate with likelihood function $L$ (4). These conjugate law depends on one or two unknown parameters.

\section{The technique of finding a set of a priori distribution laws conjugating with the observed general statistical set}

The general form of the posterior distribution $\tilde{\rho}\left(\Theta \mid x_{1}, x_{2}, \ldots, x_{n}\right)$ calculated by formula (2) is determined, accurate to the normalizing constant, only by the numerator of the right-hand side of this formula. Therefore, bellow when analyzing equalities that are accurate up to the normalizing constant, we will use the sign $\approx$. 
We describe the main steps of the technique.

Step 1: Check condition (4) for the existence of a set of a priori distributions conjugate to the likelihood function $L$.

Step 2: If the likelihood function $\mathrm{L}$ admits representation (4), then the first approximation is specified for the distribution density function $\rho_{F A}(\Theta)$ in accordance with the following rule:

A) if the estimated scalar parameter $\theta$ can (theoretically) take values on a finite interval $\left[\theta_{\min }, \theta_{\max }\right]$ or on an infinite interval from $-\infty$ to $+\infty$, then the a priori density function $\rho(\theta)$ should be considered constant on the corresponding interval, that is $\rho(\theta)=$ const at $\theta \in(-\infty ;+\infty)$;

B) if it follows from the meaning of the parameter being evaluated that it can take only positive values at $(-\infty ;+\infty)$, then we should assume that the distribution density function of the logarithm of the parameter value is constant over the entire numerical direct line, i.e. $\rho(\ln \theta)=$ const, at $\theta \in(0 ;+\infty)$.

Note that for the a priori distributions determined in this way, the well-known rule of normalizing the probability density function may be violated. However, this does not cause "technical inconvenience", since recalculation of such an "improper" a priori density function $\rho(\theta)$ into a posterior one according to formula (2) gives an ordinary density function $\tilde{\rho}\left(\theta \mid x_{1}, x_{2}, \ldots, x_{n}\right)$ that satisfies the normalization condition.

Step 3: The posteriori distribution function is recalculated using formula (2).

Note that the first recount immediately yields the corresponding form of a posterior distribution associated with the likelihood function.

Step 4: The parameters of the distribution density conjugating with the likelihood function are refined.

Let us demonstrate the implementation of the technique for the distribution laws considered above. Poisson stream. It follows from the meaning of the parameter $\theta$ that it can only take positive values, therefore, we determine

$$
\rho_{F A}(\theta) \approx \frac{1}{\theta}
$$

Then, given the fact that

$L\left(x_{1}, x_{2}, \ldots, x_{n} \mid \theta\right) \approx \theta^{\sum_{i=1}^{n} x_{i}} e^{-n \theta}$,

We have

$$
\tilde{\rho}\left(\theta \mid x_{1}, x_{2}, \ldots, x_{n}\right)=\rho_{F A}(\theta) L\left(x_{1}, x_{2}, \ldots, x_{n} \mid \theta\right) \approx \theta^{\sum_{i=1}^{n} x_{i}-1} e^{-n \theta}
$$


The right-hand side of this relation is (up to a normalizing factor not dependent on $\theta$ ) the gamma distribution density law

$$
\rho(\theta)=\frac{\beta^{\alpha}}{\Gamma(\alpha)} \cdot \theta^{\alpha-1} e^{-\beta \theta}, \theta>0
$$

with parameters $\alpha=\sum_{i=1}^{n} x_{i}$ и $\beta=n$.

Consequently, the family of conjugate a priori distributions of the parameter $\theta$ of the observed general population belongs to the class of gamma distribution laws (5).

Note that with $\alpha=1$ gamma distribution turns into an exponential distribution, with $\alpha>10$ gamma distribution approaches the normal distribution, and for large integer values $\alpha$ - into the Erlang distribution. If $\beta=1 / 2$, and the value of $\alpha$ is a multiple of $1 / 2$, then the gamma distribution coincides with the distribution of Pearson $\chi^{2}$ (chi-square).

Exponential distribution. Since $\theta>0$, we take

$$
\rho_{F A}(\theta) \approx \frac{1}{\theta}
$$

Then, given the fact that $L\left(x_{1}, x_{2}, \ldots, x_{n} \mid \theta\right) \approx \theta^{n} e^{-\left(\sum_{i=1}^{n} x_{i}\right) \theta}$ we have

$$
\tilde{\rho}\left(\theta \mid x_{1}, x_{2}, \ldots, x_{n}\right) \approx \rho_{F A}(\theta) L\left(x_{1}, x_{2}, \ldots, x_{n} \mid \theta\right) \approx \theta^{n-1} e^{-\left(\sum_{i=1}^{n} x_{i}\right) \theta} .
$$

The right-hand side of the last relation determines (up to a normalizing factor independent on $\theta$ ) the gamma distribution density (5) with parameters $\alpha=n$ and $\beta=\sum_{i=1}^{n} x_{i}$. So the set of conjugate a priori distributions of the scale parameter $\theta$ of the exponentially distributed general population belongs to the class of gamma distributions.

Uniform distribution. Since the parameter $\theta$ can take any positive values, we determine $\rho_{F A}(\theta) \approx \frac{1}{\theta}$. Then, given the fact that

$L\left(x_{1}, x_{2}, \ldots, x_{n} \mid \theta\right) \approx\left(\frac{1}{\theta}\right)^{n} \quad$ и $\quad x_{\max }(n)=\underset{1 \leq i \leq n}{\max x_{i} \leq 0,}$

we have

$$
\tilde{\rho}\left(\theta \mid x_{1}, x_{2}, \ldots, x_{n}\right) \approx \begin{cases}\rho_{F A}(\theta) \cdot L\left(x_{1}, x_{2}, \ldots, x_{n} \mid \theta\right) \approx\left(\frac{1}{\theta}\right)^{n+1}, & \theta \geq x_{\max }(n) \\ 0 \quad, \quad \theta<x_{\max }(n)\end{cases}
$$

But the right-hand side of the last relation is (up to a normalizing factor independent on $\theta$ ) the Pareto distribution density of the form 


$$
\rho(\theta) \approx \begin{cases}\frac{\alpha \theta_{\text {min }}^{\alpha}}{\theta^{\alpha+1}} & , \quad \theta \geq \theta_{\text {min }} \\ 0 & , \quad \theta<\theta_{\text {min }}\end{cases}
$$

with shape parameter $\alpha=n$ and shift parameter $\theta_{\min } \leq x_{\max }(n)$. Consequently, the set of conjugate a priori distributions of the parameter $\theta$ of a uniformly distributed random variable on $[0 ; \theta]$ belongs to the class of Pareto distributions of the form (6).

Pareto distribution. Let us consider the Pareto distribution law with an unknown value of the shape parameter $\theta$ and some fixed value of the shift parameter $x_{0}$. Since the parameter $\theta$ can take any positive values, we determine $\rho_{F A}(\theta) \approx \frac{1}{\theta}$. Then

$$
\begin{array}{r}
L\left(x_{1}, x_{2}, \ldots, x_{n} \mid \theta\right) \approx \theta^{n}\left(\frac{g_{n}}{x_{0}}\right)^{-n \theta} \cdot g_{n}^{-n} \approx \theta^{n} e^{-\left(n \ln n\left(\frac{g_{n}}{x_{0}}\right)\right) \cdot \theta}, \\
\tilde{\rho}\left(\theta \mid x_{1}, x_{2}, \ldots, x_{n}\right) \approx \rho_{F A}(\theta) \cdot L\left(x_{1}, x_{2}, \ldots, x_{n} \mid \theta\right) \approx \theta^{n-1} e^{-\left(n \ln n\left(\frac{g_{n}}{x_{0}}\right)\right) \cdot \theta} .
\end{array}
$$

The right-hand side of (8) under condition (7) determines (up to a normalizing factor independent of the parameter $\theta$ ) the gamma distribution density (5) with the parameter $\alpha=n$ and parameter

$$
\beta=n \ln n\left(\frac{g_{n}}{x_{0}}\right) \text {. }
$$

Thus, the conjugate a priori distributions of the shape parameter $\theta$ of the observed Paretodistributed general population belong to set of gamma - distribution laws.

Normal distribution law with known dispersion:

Since the parameter $\theta$ can take any positive or negative values, we determine $\rho_{F A}(\theta) \approx \theta$. Then

$$
\rho(\theta) \approx \exp \left(-\frac{n}{2 \sigma_{0}^{2}}(\bar{x}-\theta)^{2}\right)
$$

The right-hand side of formula (9) is (up to a normalizing factor independent of $\theta$ ), the density of the normal distribution with average value $\bar{x}$ and dispersion $\sigma_{0}^{2} / n$. Consequently, the family of conjugate a priori distribution laws of an unknown average value of $\theta$ a normally distributed general set (for a known variance $\sigma_{0}^{2}=D \xi$ ) itself belongs to the class of normal distribution law (9).

Normal distribution law with unknown means and unknown dispersion:

Note that for the Problem 2 the right-hand side is (up to a normalizing factor independent of $\theta$ and $h$ ) the density of two-dimensional gamma - normal distribution law : 
Since the parameter $\theta_{1}$ can take any positive or negative values, we determine $\rho_{F A}\left(\theta_{1}\right) \approx$ const . Since the parameter $\theta_{2}$ can take only positive values, we determine $\rho_{F A}\left(\theta_{2}\right) \approx \frac{1}{\theta_{2}}$. Then

$$
\rho(\theta, h) \approx\left(\lambda_{0} h\right)^{\frac{1}{2}} \cdot \exp \left(-\frac{\lambda_{0} h}{2}\left(\theta-\theta_{0}\right)^{2}\right) \cdot h^{\alpha-1} \cdot \exp (-\beta h)
$$

with parameters $\lambda_{0}=n, \theta_{0}=\bar{x}, \alpha=\frac{n-1}{2}, \quad \beta=\frac{1}{2} \sum_{i=1}^{n}\left(x_{i}-\bar{x}\right)^{2}$.

Consequently, the set of conjugate a priori distribution laws of a two-dimensional parameter belongs to the class of two - dimensional gamma-normal distribution law (10).

Method for calculating specific parameter values in conjugate a priori distribution laws.

Let we know the a priori mean values of the estimated parameter $\Theta_{0}=E \Theta=\left(E \theta_{1}, E \theta_{2}, \ldots, E \theta_{s}\right)$ and their standard deviations $\Delta_{j}=\sqrt{D \theta_{j}}, j=1,2, \ldots, s$. The parameters of the a priori distribution, as a rule, can be determined by the method of moments. We describe the implementation of this technique for the conjugate distributions considered above.

Gamma distribution. The average value $(E \theta)$ and variance $(D \theta)$ of the gamma distribution are expressed in terms of the parameters $\alpha$ and $\beta$ by the formulas $E \theta=\frac{\alpha}{\beta}, D \theta=\frac{\alpha}{\beta^{2}}$. Substituting in these relations instead of $E \theta$ and $D \theta$ respectively, the given values of $\theta_{0}$ and $\Delta^{2}$, we obtain the expressions for $\alpha$ and $\beta$ :

$$
\alpha=\frac{\theta_{0}^{2}}{\Delta^{2}}, \quad \beta=\frac{\theta_{0}}{\Delta^{2}} .
$$

Pareto distribution. The shape parameter $\alpha$ and the shift parameter $\theta_{\min }$ can be found from the given values $\theta_{0}=E \theta$ and $\Delta^{2}=D \theta$ from the solution of the system of equations

$$
\left\{\begin{array}{c}
E \theta=\frac{\alpha \theta_{\min }}{\alpha-1}=\theta_{0} \\
D \theta=\frac{\alpha \theta_{\min }^{2}}{(\alpha-1)^{2}(\alpha-2)}=\Delta^{2}
\end{array}\right.
$$

The solution to has the form:

$$
\alpha=1+\sqrt{1+\frac{\theta_{0}^{2}}{\Delta^{2}}} \quad, \quad \theta_{\min }=\frac{1}{\alpha} \theta_{0}(\alpha-1) .
$$


Using as a priori laws the probability distributions associated with the observed general population allows us to determine their general form, i.e., it defines a whole set of a priori distribution laws $\{\rho(\Theta ; D)\}$. However, when implementing the Bayesian approach, we must operate with a specific a priori distribution law, which requires knowledge of the numerical values $D_{0}$ of the parameters D.

Since the calculation of parameters for the normal law with unknown means and known dispersion is obviously, we describe the procedure for calculating parameters only for the normal law with unknown means and unknown dispersion. From the properties of two-dimensional gamma - normal distribution law it follows that the partial a priori distribution of a parameter $h$ is a gamma distribution law with parameters $\alpha$ and $\beta$. Therefore, using the given values of $h_{0}=E h$ and $\Delta_{h}^{2}=D h$, we compose two equations with respect to $\alpha$ and $\beta: E h=\alpha / \beta=h_{0}, D h=\alpha / \beta^{2}=\Delta_{h}^{2}$ . Then we obtain the solution: $\alpha=h_{0}^{2} / \Delta_{h}^{2}$ and $\beta=h_{0} / \Delta_{h}^{2}$.

The value of the shear parameter in the distribution (6) is equal to $\theta_{0}$ and

$$
\Delta_{\theta}^{2}=\frac{\beta}{\lambda_{0} \alpha} \cdot \frac{\alpha}{\alpha-1}, \quad \lambda_{0}=\frac{1}{\Delta_{\theta}^{2}} \cdot \frac{\alpha}{\alpha-1} .
$$

Recalculation of parameter values during the transition from a priori distribution to a posterior distribution.

Let $\{\rho(\Theta ; D)\}$ be a set of a priori distributions conjugate to the likelihood function $L\left(x_{1}, x_{2}, \ldots, x_{n} \mid \Theta\right)$ of the observations $D\left(d_{1}, d_{2}, \ldots, d_{q}\right)$ and let we have $\left(D\left(d_{1}, d_{2}, \ldots, d_{q}\right)\right.$ - vector of parameters on which the conjugate a priori distribution $\rho(\Theta ; D)$ depends. And let $D_{0}$ is the given (known) parameter values $D$ in the analyzed case. Then, using a series of identical transformations the right side of the ratio $\tilde{\rho}\left(\Theta \mid x_{1}, x_{2}, \ldots, x_{n}\right) \approx \rho\left(\Theta ; D_{0}\right) \cdot L\left(x_{1}, x_{2}, \ldots, x_{n} \mid \Theta\right)$ is given, up to factors independent on $\Theta$, to the form $\rho\left(\Theta ; D\left(x_{1}, x_{2}, \ldots, x_{n}\right)\right.$. Here the last function belongs to the set $\rho(\Theta ; D)$, and each of the components $d_{j}\left(x_{1}, x_{2}, \ldots, x_{n}\right),(j=1,2, \ldots, q)$ of the parameter vector $D\left(x_{1}, x_{2}, \ldots, x_{n}\right)$ are a function of $D_{0}$ and $\left(x_{1}, x_{2}, \ldots, x_{n}\right)$.

Let us describe the implementation of this general scheme for the distribution laws considered above.

Poisson distribution. As we saw earlier, the likelihood function of the observations of the Poisson population is:

$$
L\left(x_{1}, x_{2}, \ldots, x_{n} \mid \theta\right) \approx \theta^{\sum_{i=1}^{n} x_{1}} e^{-n \cdot \theta} .
$$


In view of (13), using the gamma distribution (5) as the a priori distribution of the parameter, we have:

$$
\rho\left(\theta \mid x_{1}, x_{2}, \ldots, x_{n}\right) \approx \theta^{\alpha-1} e^{-\beta \theta} \cdot \theta^{\sum_{i=1}^{n} x_{1}} e^{-n \cdot \theta}=\theta^{\alpha+\sum_{i=1}^{n} x_{1}-1} e^{-(\beta+n) \cdot \theta} .
$$

This confirms that the conjugacy of the a priori gamma distribution, and the posterior gamma distribution is determined by the parameters

$$
\tilde{\alpha}=\alpha+\sum_{i=1}^{n} x_{1}, \quad \tilde{\beta}=\beta+n .
$$

Exponential distribution. Using the gamma distribution (5) as the a priori distribution of the parameter, we have:

$$
\tilde{\rho}\left(\theta \mid x_{1}, x_{2}, \ldots, x_{n}\right) \approx \theta^{\alpha-1} e^{-\beta \theta} \cdot \theta^{n} e^{-\left(\sum_{i=1}^{n} x_{1}\right) \theta}=\theta^{\alpha+n-1} e^{-\left(\beta+\sum_{i=1}^{n} x_{1}\right) \cdot \theta} .
$$

We see that the posterior distribution of the parameter again obeys the gamma distribution law (5), but with the parameters

$$
\tilde{\alpha}=\alpha+n \quad, \quad \tilde{\beta}=\beta+\sum_{i=1}^{n} x_{1}
$$

Uniform distribution. Using the Pareto distribution (6) as the a priori distribution of the parameter, we have:

$$
\tilde{\rho}\left(\theta \mid x_{1}, x_{2}, \ldots, x_{n}\right) \approx \frac{\alpha \theta_{\min }^{\alpha}}{\theta^{\alpha+1}} \cdot \frac{1}{\theta^{n}} \quad \text { при } \theta \geq \max \left\{\theta_{\min } ; x_{1}, x_{2} . ., x_{n}\right\} .
$$

It follows that the posterior distribution of the parameter $\theta$ is described, as well as the a priori distribution, by the Pareto law, but with the parameters:

$$
\tilde{\alpha}=\alpha+n \quad, \quad \tilde{\theta}_{\min }=\max \left\{\theta_{\min } ; x_{1}, x_{2}, \ldots, x_{n}\right\} .
$$

Pareto distribution. Using the gamma distribution (5) as the a priori distribution of the parameter $\theta$, we have:

$$
\rho\left(\theta \mid x_{1}, x_{2}, \ldots, x_{n}\right) \approx \theta^{\alpha-1} e^{-\beta \theta} \theta^{n} e^{-\left(n \ln \left(\frac{g_{n}}{x_{0}}\right)\right) \theta}=\theta^{\alpha+n-1} e^{-\left(\beta+n \ln \left(\frac{g_{n}}{x_{0}}\right)\right) \theta} .
$$

It can be seen that the posterior distribution of the parameter $\theta$ again obeys the gamma distribution law (5), but with the parameters

$$
\tilde{\alpha}=\alpha+n \quad, \quad \tilde{\beta}=\beta+n \ln \left(\frac{g_{n}}{x_{0}}\right) .
$$


Let $\{\rho(\Theta ; D)\}$ be a set of a priori distribution laws associated with the likelihood function $L\left(x_{1}, x_{2}, \ldots, x_{n} \mid \Theta\right)$ of our observations; $D\left(d_{1}, d_{2}, \ldots, d_{q}\right)$ - the vector of parameters on which the conjugate a priori distribution $\rho(\Theta ; D)$ depends; $D_{0}$ - given (known) values of the parameters $D$ in the analyzed case.

Normal law with known dispersion. $d_{1}=\theta_{0}, d_{2}=\Delta_{0}^{2}$,

$$
\begin{gathered}
\tilde{d}_{1}\left(x_{1}, x_{2}, \ldots, x_{n}\right)=E\left(\theta \mid x_{1}, x_{2}, \ldots, x_{n}\right)=\left(\frac{1}{\sigma_{0}^{2} / n} \cdot \bar{x}+\frac{1}{\Delta_{0}^{2}} \cdot \theta_{0}\right) /\left(\frac{1}{\sigma_{0}^{2} / n}+\frac{1}{\Delta_{0}^{2}}\right), \\
\tilde{d}_{2}\left(x_{1}, x_{2}, \ldots, x_{n}\right)=D\left(\theta \mid x_{1}, x_{2}, \ldots, x_{n}\right)=\left(\frac{1}{\sigma_{0}^{2} / n}+\frac{1}{\Delta_{0}^{2}}\right)^{-1} .
\end{gathered}
$$

Note that the average value $\tilde{d}_{1}$ and dispersion $\tilde{d}_{2}$ of a posterior normal distribution law are the weighted average values of a priori and sample mean and variances, respectively.

Normal law with known dispersion. When implementing the general scheme for converting a priori parameters into a posteriori parameters ones in this case, one should take into account the representation of the likelihood function $L$ in the form (6); a priori density form of twodimensional gamma - normal distribution (10) too. And the vector of parameters is $D_{0}=\left(\lambda_{0} ; \theta_{0} ; \alpha ; \beta\right)$;

$$
\left.\tilde{\lambda}_{0}=\lambda_{0}+n, \quad \tilde{\theta}_{0}=\frac{\lambda_{0} \theta_{0}+n \bar{x}}{\lambda_{0}+n}, \quad \tilde{\alpha}=\alpha+\frac{n}{2}, \quad \tilde{\beta}=\beta+\frac{1}{2} \sum_{i=1}^{n}\left(x_{i}-\bar{x}\right)\right)^{2}+\frac{1}{2}\left(\bar{x}-\theta_{0}\right)^{2} /\left(\frac{1}{n}+\frac{1}{\lambda_{0}}\right) \text {. }
$$

\section{RESULTS}

Task 1. The intensity of the flow entering the recording element of the measuring instrument per second is described by the Poisson distribution with an unknown parameter value $\theta=E \xi$. The number of registered particles $x_{i}$ per second is shown in the table 1:

Table1. The number of registered particles per second

\begin{tabular}{|c|l|l|l|l|l|l|l|l|}
\hline$i$ & 1 & 2 & 3 & 4 & 5 & 6 & 7 & 8 \\
\hline$X i$ & 3 & 1 & 4 & 2 & 6 & 3 & 3 & 2 \\
\hline$\theta=E \xi$
\end{tabular}

From the results of the analysis of the operation of similar measuring instruments in similar conditions, a priori mean value $\theta_{0}=E \theta=3,6$ and variance $\Delta_{0}^{2}=D \theta=0,09$ are known.

Decision. In this case, the conjugate a priori distribution of the parameter $\theta$ exists and is described by the gamma law. The parameters $\alpha$ and $\beta$ of gamma distribution are determined from the solution of the system of equations:

$$
\left\{\begin{array}{c}
E \theta=\frac{\alpha}{\beta}=3,6 \\
D \theta=\frac{\alpha}{\beta^{2}}=0,09
\end{array}\right.
$$


Solving this system we get $\alpha=144$ and $\beta=40$.

In accordance with the above recalculation formulas (12) for the gamma distribution, we have: $\tilde{\alpha}=144+24=168, \tilde{\beta}=40+8=48$.

Thus $\hat{\theta}^{(5)}=E\left(\theta \mid x_{1}, x_{2}, \ldots, x_{n}\right) \approx \tilde{\alpha} / \tilde{\beta}=3,5$ and it can be argued that inequalities $\gamma_{0,025}(\tilde{\boldsymbol{\alpha}}, \widetilde{\boldsymbol{\beta}})<\theta<\gamma_{0,975}(\widetilde{\boldsymbol{\alpha}}, \widetilde{\boldsymbol{\beta}})$ are true with probability $P_{0}=0,95$, where $\gamma_{q}(\widetilde{\boldsymbol{\alpha}}, \widetilde{\boldsymbol{\beta}})$ is the $100 \mathrm{q} \%$ - gamma distribution point with the parameters $\widetilde{\boldsymbol{\alpha}}$ and $\widetilde{\beta}$. Using the Excel function ГАММАРАСПР, we get $\widetilde{\theta}^{(Б)} \in[2,99 ; 4,05]$ with probability $P_{0}=0,95$. Note that the ГАММАРАСПР function built into Excel differs from the standard table function that defines the gamma distribution in that instead of the input parameter $\boldsymbol{\beta}$, you must specify the value inverse to it $1 / \beta$.

Relevant estimates using the maximum likelihood method: $\hat{\theta}_{M L M}=\bar{x}=3,0$ and $\theta_{M L M} \in[1,80 ; 4,20]$ with probability $P_{0}=0,95$.

Thus, the use of a priori information on the parameter $\theta$ in allowed us to narrow the range of the interval estimate by more than two times.

Task 2. In some cases of metrological practice, the measurement results or their errors are considered as random variables $\xi$ uniformly distributed over a certain interval $[0 ; \theta]$. Let the uniformly distributed random variable take the values: $x_{1}=1,2 ; x_{2}=2,5 ; x_{3}=0,5$; $x_{4}=3,2 ; x_{5}=2,9$. Let the parameter $\theta$ be assumed to be unknown. A priori information: the average value of the parameter $\theta=5$ and the standard deviation is $\sigma=1,4$.

Decision. In this case, the conjugate a priori distribution of the parameter $\theta$ exists and is described by the Pareto distribution with the shape parameter $\alpha$ and the shift parameter $\theta_{\text {min }}$, which are determined according to (10):

$$
\alpha=1+\sqrt{1+\frac{\theta_{0}^{2}}{\Delta^{2}}}=1+\sqrt{1+\frac{5^{2}}{1,4^{2}}}=4,71, \quad \theta_{\min }=\frac{1}{\alpha} \theta_{0}(\alpha-1)=\frac{1}{4,71} \cdot 5 \cdot 3,71=3,94 .
$$

The parameters of the posterior Pareto distribution are determined by the conversion formulas (14):

$$
\tilde{\alpha}=\alpha+n=4,71+5=9,71, \quad \tilde{\theta}_{\min }=\max \left\{\theta_{\min }, x_{1}, x_{2}, \ldots, x_{5}\right\}=3,94
$$

Respectively: 


$$
\hat{\theta}^{(B)}=E\left(\theta \mid x_{1}, x_{2}, \ldots, x_{5}\right) \approx \frac{\tilde{\alpha} \cdot \tilde{\theta}_{\min }}{\tilde{\alpha}-1}=4,39 \text { and } \theta \in\left[\theta_{0,975}\left(\tilde{\alpha} ; \tilde{\theta}_{\min }\right) ; \theta_{0,025}\left(\tilde{\alpha} ; \tilde{\theta}_{\min }\right)\right]
$$

with probability $P_{0}=0,95$, where $\theta_{q}\left(\tilde{\alpha} ; \tilde{\theta}_{\text {min }}\right)$ is the $100 \mathrm{q} \%$ Pareto distribution point with parameters $\tilde{\alpha}$ and $\tilde{\theta}_{\min }$. Since the distribution function Pareto is determined by the relation

$$
F(\theta)=P\left\{\xi\left(\tilde{\alpha}, \tilde{\theta}_{\min }\right)<\theta\right\}=1-\left(\frac{\tilde{\theta}_{\min }}{\theta}\right)^{\tilde{\alpha}},
$$

then the values $\theta_{0,025}\left(\tilde{\alpha} ; \tilde{\theta}_{\min }\right)$ and $\theta_{0,975}\left(\tilde{\alpha} ; \tilde{\theta}_{\min }\right)$ can be determined from the equations:

$$
\left(\frac{\tilde{\theta}_{\min }}{\theta_{0,025}\left(\tilde{\alpha} ; \tilde{\theta}_{\max }\right)}\right)^{\tilde{\alpha}}=0,025 \text { и }\left(\frac{\tilde{\theta}_{\min }}{\theta_{0,975}\left(\tilde{\alpha} ; \tilde{\theta}_{\min }\right)}\right)^{\tilde{\alpha}}=0,975 \text {. }
$$

The solution to these equations has the form:

$$
\theta_{1}=\theta_{0,975}\left(\tilde{\alpha} ; \tilde{\theta}_{\min }\right)=\left(\frac{\tilde{\theta}_{\min }}{0,025^{1 / \tilde{\alpha}}}\right)=3,95, \quad \theta_{2}=\theta_{0,025}\left(\tilde{\alpha} ; \tilde{\theta}_{\min }\right)=\left(\frac{\tilde{\theta}_{\min }}{0,975^{1 / \tilde{\alpha}}}\right)=5,76 \text {. }
$$

Then $\tilde{\theta}^{(5)} \in[3,95 ; 5,76]$ with probability $P_{0}=0,95$.

Statistical estimates constructed using the maximum likelihood method are: $\hat{\theta}_{M L M}=\frac{n+1}{n} \cdot \max \left\{x_{1}, x_{2}, \ldots, x_{5}\right\}=\frac{6}{5} \cdot 3,2=3,84 \quad ; \quad \hat{\theta}_{M L M} \in[3,22 ; 6,69] \quad$ with probability $P_{0}=0,95$. Note that the above estimate $\hat{\theta}_{M L M}$ was corrected for unbiasedness.

Task 3a. Suppose that we have the following information about the analyzed data: (a) The statistical data is distributed normally with an unknown average value $\theta$ and known dispersion $\sigma_{0}^{2}=0,28$;

(b) There are measurement results of $n=10$ randomly selected values of the measured parameter $y$ (see Table 2).

Table 2. Statistical data.

\begin{tabular}{|c|c|c|c|c|c|c|c|c|c|c|}
\hline$i$ & 1 & 2 & 3 & 4 & 5 & 6 & 7 & 8 & 9 & 10 \\
\hline$X i$ & 0,54 & 1,20 & 0,36 & 0,80 & 0,42 & 2,10 & 0,70 & 0,25 & 0.90 & 0,48 \\
\hline
\end{tabular}

(c) Let from history and from results of previous measurements it is known that the a priori values of the mean $E \theta=\theta_{0}=0,60$ and dispersion $D \theta=\Delta_{0}^{2}=0,03$. It is required, using the conjugate a priori distribution of the parameter, to obtain Bayesian point and interval (with confidence level $\left.P_{\theta}=0,95\right)$ estimates of the average value of a random variable and compare them 
with the corresponding estimates obtained by MLM.

Task $3 b$. In the conditions of Task $3 a$ we assume that the a priori value of the variance $\sigma_{0}^{2}$ (accuracy parameter $p=1 / \sigma_{0}^{2}$ ) is unknown. It is required, using the conjugate a priori distribution of the parameters $\theta$ and $h$, to obtain a Bayesian point estimate and construct the corresponding confidence (with a confidence level $P_{\theta}=0,95$ ) estimates of the average value and standard deviation of the quantity and compare them with the corresponding estimates of the MLM. Also build two-dimensional confidence areas corresponding to confidence levels $P_{(\theta, h)}=0,95$ and $P_{(\theta, h)}=0,98$.

Table 3 shows the point estimates and confidence intervals based on the Bayesian approach and the MLM.

Table 3. Comparison of the Bayesian approach and the maximum likelihood method

\begin{tabular}{|c|c|c|c|c|c|c|c|c|}
\hline & \multicolumn{4}{|c|}{ Bayesian approach } & \multicolumn{4}{|c|}{ Maximum likelihood method } \\
\hline & \multicolumn{2}{|c|}{ Average value } & \multicolumn{2}{|c|}{ Dispersion } & \multicolumn{2}{|c|}{ Average value } & \multicolumn{2}{|c|}{ Dispersion } \\
\hline $\begin{array}{c}\text { Ex } \\
\mathrm{a} \\
\mathrm{m} \\
\mathrm{pl} \\
\mathrm{e} \\
\text { № }\end{array}$ & $\begin{array}{l}\text { The } \\
\text { mathe } \\
\text { matic } \\
\text { al } \\
\text { expec } \\
\text { tation } \\
\text { of the } \\
\text { avera } \\
\text { ge } \\
\text { value }\end{array}$ & $\begin{array}{l}\text { Confidence } \\
\text { Interval of the } \\
\text { average value }\end{array}$ & $\begin{array}{c}\text { The } \\
\text { mathema } \\
\text { tical } \\
\text { expectati } \\
\text { on of the } \\
\text { dispersio } \\
n\end{array}$ & $\begin{array}{l}\text { Confidence } \\
\text { Interval of } \\
\text { dispersion }\end{array}$ & $\begin{array}{l}\text { The } \\
\text { mathe } \\
\text { matical } \\
\text { expecta } \\
\text { tion of } \\
\text { the } \\
\text { average } \\
\text { value }\end{array}$ & $\begin{array}{l}\text { Confidence } \\
\text { Interval of the } \\
\text { average value }\end{array}$ & $\begin{array}{l}\text { The } \\
\text { mathe } \\
\text { matical } \\
\text { expect } \\
\text { ation } \\
\text { of the } \\
\text { dispers } \\
\text { ion }\end{array}$ & $\begin{array}{l}\text { Confidence } \\
\text { Interval of } \\
\text { dispersion }\end{array}$ \\
\hline 1 & 0,691 & {$[0,451 ; 0,931]$} & 0,0145 & - & 0,775 & {$[0,447 ; 1,103]$} & 0,03 & - \\
\hline 2 & 0,697 & {$[0,363 ; 1,187]$} & 0,14 & {$[0,1 ; 0,441]$} & 0,775 & {$[0,363 ; 1,187]$} & 0,297 & {$[0,088 ; 0,528]$} \\
\hline
\end{tabular}

It can be seen that the application of the Bayesian approach allows one to construct more accurate and reliable estimates. Figure 2 shows a general view of gamma - normal distribution law. 


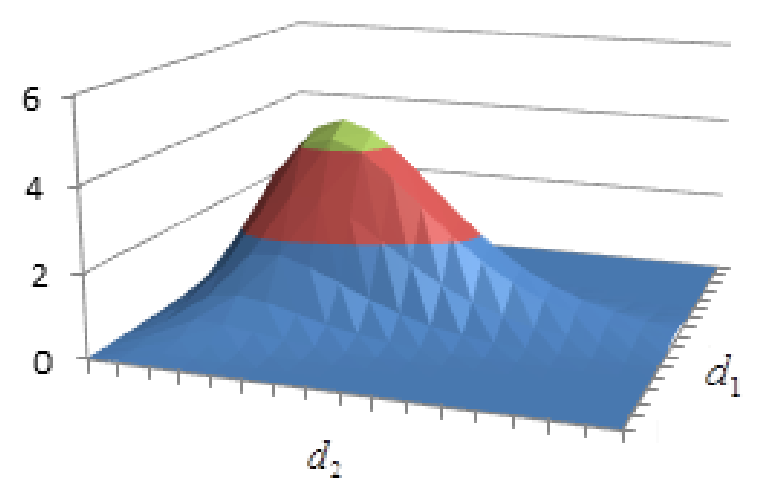

Figure 2. General view of gamma - normal distribution.

Figure 3 shows confidence areas (in the plane of parameters $(\theta ; h)$ - accuracy and average value) for confidence probabilities $P_{(\theta ; h)}=0,95$ and $P_{(\theta ; h)}=0,975$. It can be seen that the areas are dropshaped. These confidence areas are level lines of the function of two variables $\rho(\theta ; h)$ by the plane $\rho=$ const . The values of const are selected in such a way that the volume of the upper cut-off part of the figure that sets the distribution density $\rho(\theta ; h)$ is 0,95 and 0,975 , respectively. Note that with increasing $n$ confidence areas will become more and more similar to ellipses, since the gamma normal distribution will tend to a two-dimensional normal law. We also note that currently in the scientific works of other authors the methods for constructing confidence regions which have the shapes of ellipses, rectangles, ellipsoids are described and implemented.

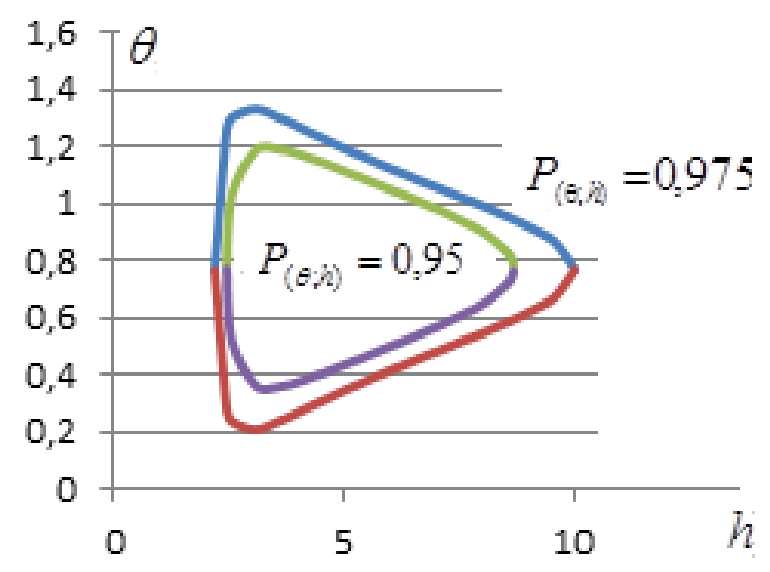

Figure 3. Confidence area in the plane "average value-accuracy".

Thus, results of simulation demonstrate that the Bayesian approach made it possible to narrow the confidence interval by 1.9 times in comparison with the maximum likelihood method.

\section{DISCUSSIONS}

Modern innovative projects lead to the need to develop new measurement technical means and devices with specified technical, metrological and operational characteristics. The above characteristics of a new creating product are detailed in the relevant technical specifications of the 
development of a new product. Before the introduction of these technical means and devices or before the state acceptance of these technical means and devices, a whole test cycle is carried out. The goal of testing these devices is to confirm the specified characteristics defined in the technical projects. To achieve this goal, the results of testing are carefully analyzed, including processed by statistical methods.

The article presents a method for constructing statistical estimates of the distribution parameters of random variables for the following laws: Poisson law, exponential law, uniform law, Pareto law and normal law. The presented results, cover a fairly wide variety of distribution laws encountered in the practice of solving measurement problems, in the practice of solving problems of increasing the quality of construction materials and in the practice of working the results of testing of measuring devices.

When constructing statistical estimates based on the Bayesian approach, distributions conjugating with the observed general statistical set play an important role. The article formulates the necessary conditions for the existence of conjugate distributions. An algorithm for calculating the unknown parameters of the above distribution laws, as well as an algorithm for calculating the unknown parameters of the conjugate distributions are described.

The Bayesian approach can provide significant gains in accuracy with limited sample sizes and numbers compared to the traditional maximum likelihood method. This circumstance makes the proposed method especially effective in the tasks of evaluating the metrological characteristics of measuring complexes and measuring instruments, in the case when repeated repetition of tests seems burdensome or impossible. In the case when we are able to increase the volume and number of sampling data, both approaches as Bayesian approach as MLM will give ever closer results.

Using a priori information about an unknown parameter (unknown parameters) allows us to construct effective statistical estimates. In the examples considered in the paper the Bayesian scientific approach allowed us to halve the range of confidence intervals in comparison with the classical MLM.

The results obtained in the article can find application in the development of measurement methods, in the verification and calibration of measuring instruments, in the development of practical methods for identifying systematic errors and so on.

The algorithms and results obtained in the article are aimed at methodological support of the problems described above. The algorithms are based on taking account the available statistical data together with a priori information about the process or object under study.

The developed method can be used in order to create self-learning and self-tuning systems. For this purpose, it is necessary to consistently apply relevant recalculation formulas.

\section{CONCLUSION}


The results of applying the Bayesian approach to the construction of statistical estimates of the parameters of the distribution laws that are most often encountered in solving problems of metrological support of measuring instruments are described.

The effectiveness of the methods is demonstrated by four examples showing that the range of the confidence interval can be reduced by two or more times.

The Bayesian approach presented in this article was realized as the module of the software package that was developed by author with using Matlab software. The developed software package allows to construct effective statistical parameter estimates for the distribution laws considered in the article.

\section{CONFLICT OF INTEREST}

The authors confirm that the information provided in the article does not contain a conflict of interest.

\section{REFERENCES}

Ayvazyan, S.A. (2008). Bayesian approach in econometric analysis. Applied Econometrics, 1(9), 93-130. Duyguİçen, D. (2019). A new approach for probability calculation of fuzzy events in Bayesian Networks. International Journal of Approximate Reasoning, 108, 76-88.

Francisco, S., Guzmán, J., Rosa, B., Rodríguez, C., Doimeadios, M., \& Ángel, R. (2019). Analytical metrology for nanomaterials. Present achievements and future challenges. Analytica Chimica Acta, 1059, 1-15.

Gao, W., Haitjema, H., Fang, F., Leach, R., Cheung, C., Savio, E., \& Linares, J. (2019). On-machine and in-process surface metrology for precision manufacturing. CIRP Annals, 68, 2.

Geweke, J., \& Durham, G. (2019). Sequentially adaptive Bayesian learning algorithms for inference and optimization. Journal of Econometrics, 210(1), 4-25.

Higgins, V., Asgari, S., \& Adeli, K. (2019). Choosing the best statistical method for reference interval estimation. Clinical Biochemistry, 71, 14-16.

Khayrullin R.Z., Kornev A.S., Kostoglotov A.A., Lazarenko S.V. A.S.(2021). Mathematical simulation of decision error functions in tolerance control of the performance of measuring techniques. Measurement Techniques, 63(9), 680-685.

Khayrullin, R.Z. (2019). Method for constructing estimates of accuracy of measuring equipment based on Bayesian scientific approach. E3S Web of Conferences 135, 01070. ITESE. https://doi.org/10.1051/ e3sconf / 201913501070 https://scientist-view.ru.

Ković, M., \& Butenweg, C. (2019). Innovative decoupling system for the seismic protection of masonry infill walls in reinforced concrete frames. Engineering Structures, 197, 109435.

Kropotov, D., \& Vetrov, D. (2007). On one method of non-diagonal regularization in sparse Bayesian learning. Proc. of 7 -th Open German-Russian Workshop on Pattern Recognition and Image Understanding. 
Kuznetsov, V.A., Isaev, L.K., \& Shayko, I.A. (2005). Metrology. Moscow. Standartinform. 298 p.

Lavrik, E., Frankenfeld, U., Mehta, S., Panasenko, I., \& Schmidt, H. (2019). High-precision contactless optical 3Dmetrology of silicon sensors Nuclear Instruments and Methods in Physics Research. Section A: Accelerators, Spectrometers, Detectors and Associated Equipment, 935, 167-172.

Lazarenko S.V., Kostoglotov A.A. ,Khayrullin R.Z., Kornev A.S. (2021).Intelligent Regularized Measurement Procedure Based on the Use of a Dynamic Model of the Combined Maximum Principle and the Theory of Regularization. Dynamics of Technical Systems (DTS 2020). IOP Conf. Series: Materials Science and Engineering 1029 (2021) 012083.

measuring instruments, taking into account the periodicity of verification. Measuring Techniques 4, 22-27.

Michael, D., Thomas, A., \& Adcock, A. (2018). Prediction of tidal currents using Bayesian machine learning. Ocean Engineering, 158, 221-231.

Mishchenko V.I., Kravtsov A.N., Mamleev T.F. (2021). Semi-Markov model of functioning of redundant.

Touzani, S., Ravache, B., Crowe, E., \& Granderson, J. (2019). Statistical change detection of building energy consumption. Applications to savings estimation. Energy and Building Journal, 18(515),123-136.

Vishnyakov B. V., \& Egorov A. I. (2013). Construction of confidence areas for the trajectories of objects in the problems of machine vision. Proceedings of the RAS. Theory and control systems, 3, 124-132.

Volchkov, A.A., Isaev, Yu.A., Leonova, K.S., Fufaeva, O.A., \& Khayrullin, R.Z. (2019). The method of constructing estimates of the accuracy of measurements based on the use of posterior information. Bulletin of the metrologist, 4 , $18-21$.

Wang, L., Zhao, L., Yu, L., Wang, J., \& Bi, G. (2019). Structured Bayesian learning for recovery of clustered sparse signal. Signal Processing, 166, 107255.

Yang, H., Jintao, Ke.J., \& Jieping, Ye.J. (2018). A universal distribution law of network detour ratios. Transportation Research. Part C: Emerging Technologies, 96, 22-37.

Yochan, K., \& Jinkyun, P. (2019). Incorporating prior knowledge with simulation data to estimate PSF multipliers using Bayesian logistic regression. Reliability Engineering \& System Safety, 189, 210-217. 